\title{
CARTESIAN HARMONIC POLYNOMIALS FOR A PROBLEM OF DEFORMATION OF A LONG, CURRENT-CARRYING ELASTIC CYLINDRICAL CONDUCTOR OF NEARLY-CIRCULAR NORMAL CROSS-SECTION
}

\author{
S.M. El Sheshtawy', A.-S.F. Obada1, M.S. Abou-Dina ${ }^{2}$, A.F. Ghaleb ${ }^{2 *}$ \\ 1- Department of Mathematics, Faculty of Science, (Boys) Al-Azhar University, Nasr City 11884, Cairo, Egypt \\ 2 - Department of Mathematic, Faculty of Science, Cairo University, Giza 12613, Egypt \\ * Corresponding Author: afghaleb@sci.cu.edu.eg
}

\begin{abstract}
We use a method recently introduced by some of the authors to obtain a semi-analytical solution to a problem of deformation of a long cylindrical conductor carrying a steady axial current, in the quasi-electrostatic approximation. The method relies on the expansion of the unknown harmonic functions arising in the process of solution in terms of Cartesian polynomial and rational harmonic functions. The normal cross-section of the conductor is taken to be nearly circular and the thermal and magnetic parts are solved independently of each other and of the elastic problem.

Numerical results and plots are provided and discussed. A comparison with the case of a circular crosssection allows to assess the influence of imperfection of the cross-sectional shape on the quantities of practical interest. The effect of the dependence of the magnetic permeability on strain is investigated as well.
\end{abstract}

Keywords: Thermo-magnetoelasticity, Cartesian polynomials, Plane problem, Electric conductor, Long cylinder, Nearly- circular cross-section, Magnetic vector potential, Semi-analytical solution.

\section{INTRODUCTION}

The interactions between the mechanical, thermal and electromagnetic fields in deformable solids have been the subject of permanent interest to researchers and engineers in the past hundred years or so. This is conditioned by the important applications in technology, especially in those devices which contain current-carrying parts. Important contributions to the theory and applications of this subject in Technology and in Geophysics may be found in many books, among which we cite only [1], [2] and [3]. The applications in electromagnetic elasticity span a wide variety of devices like sensors, actuators, magnetic levitation, etc.

An important application of the theory of electromagnetic continua concerns the deformation of cylindrical conductors carrying a steady axial current. Such elements are essential in electrical stations under the name of "busbars". These are usually of rectangular or cylindrical normal cross-section, tens of centimeters long and carry currents of several ampères. It is expected that a respectable amount of heat is released from these busbars, which is nothing else but the well-known Joule heat. In addition, the busbar statically deforms under the action of heat and the magnetic forces due to the electric current. The problem thus involves mechanical, thermal and magnetic interactions, and requires a suitable mathematical model to describe them. In the quasi-electrostatic, uncoupled approach to be adopted here, it is convenient to use the magnetic vector potential which is parallel to the axis of the cylinder. The mathematical problem reduces to the determination of five harmonic functions, interrelated by a set of boundary conditions: Two of these harmonic functions combine to yield Airy's stress function in the cross-sectional domain in case the mechanical problem is solved in stresses. A third harmonic function enters in the formulation of the thermal problem for temperature distribution. The two remaining harmonic functions are used to describe the vector potential distribution inside the conductor and in the surrounding region. The results find application in 
calculating the deformation of straight central sections of electric conductors in various instruments, in particular busbars in electric power stations, and the stresses induced in them due to the electric current.

The deformation of long, current-carrying wires has been the subject of active research for fifty years or so. We only cite the following references by Ghaleb [4] for the circular boundary, Ayad [5] for the elliptical boundary, Abou-Dina and Ghaleb [6] for a boundary integral formulation of the problem, Deviatkin [7] for cylinders of elliptic or narrow rectangular cross-sections, El Dhaba et al. $[8,9,10]$ for the elliptic or square boundaries by boundary integrals, including a numerical approach, El Dhaba [11] and El Dhaba and Ghaleb [12] for a rod with square normal cross-section in an initially uniform transverse magnetic field by boundary integrals. Recently, the authors have investigated the elliptic and the rectangular contours under the Dirichlet thermal condition and uniform normal extension on the boundary [13]. Other work relating to the mechanical-thermal-electromagnetic interaction may be found in the literature in a different context. We cite, among others, the work by Othman and Abbas [14] and Altenbach et al. [15].

The present work concentrates on the cylindrical conductor of nearly-circular normal cross-section as being an imperfection of the circular one. The aim is to investigate the influence of this imperfection on the distribution of quantities of practical interest on the cross-sectional contour, viz. the magnetic field and the stresses. The results may be of interest in the non-destructive testing of materials. Moreover, the model includes two material constants illustrating the dependence of the magnetic permeability on the strain. These will go into the equations of elasticity. We believe that proper elastic measurements may lead to interesting relations which would permit the determination of these two constants (c.f. [4]).

The method of solution relies on the expansion of the unknown harmonic functions of the problem in Cartesian polynomials and rational harmonic functions. The coefficients of these expansions are determined numerically using the method of boundary collocation.

The basic equations and accompanying conditions are presented in brief, more details may be found in [13]. Numerical results are provided and discussed.

\section{PROBLEM FORMULATION}

Let the long elastic cylinder carrying a steady, axial uniform electric current of density $J$ be placed in a medium of steady temperature $T_{e}$. A system of orthogonal Cartesian coordinates $(x, y, z)$ with $\mathrm{z}$-axis along the axis of the cylinder and centered at point 0 as shown on Fig. 1 is used to describe the problem, where we have indicated the directions of the electric current density and the magnetic vector potential. The normal cross-section is denoted ${ }^{D}$ with boundary $C$.

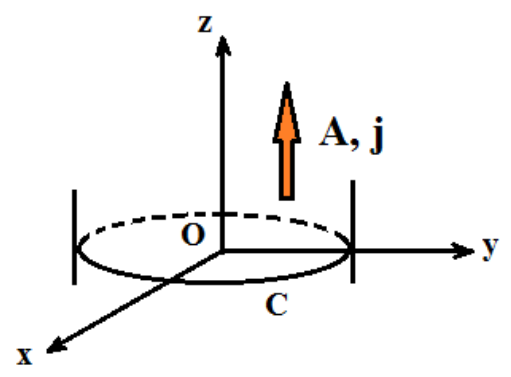

Figure 1: Geometry of the problem.

The cross-section $\mathrm{C}$ has parametric equations: 


$$
\mathrm{x}=\mathrm{x}(\theta), \quad \mathrm{y}=\mathrm{y}(\theta),
$$

where ${ }^{\theta}$ is the usual polar angle measured from the origin of coordinates. The unit vectors along the normal and the tangent to the contour $\mathrm{C}$ with the usual orientation are:

$$
\mathrm{n}=\left(\frac{\dot{\mathrm{y}}}{\omega},-\frac{\dot{\mathrm{x}}}{\omega}\right), \quad \tau=\left(\frac{\dot{\mathrm{x}}}{\omega}, \frac{\dot{\mathrm{y}}}{\omega}\right) .
$$

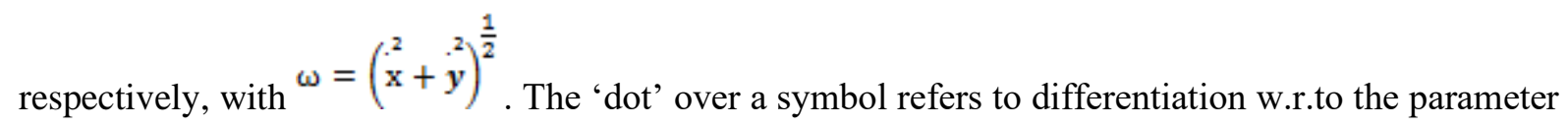
$\theta$. When the boundary parameter in the arc length, then $\omega=1$. One can use formula (2) to find the normal and the tangential derivatives of a function $\mathrm{f}(\mathrm{x}, \mathrm{y})$ on the boundary.

\section{BASIC FIELD EQUATIONS AND BOUNDARY CONDITIONS}

The governing equations of static, linear uncoupled thermo-magnetoelasticity are quoted here below without proof as presented in [10]. These will be solved under prescribed proper mechanical and thermal boundary conditions. The model takes into account the dependence of the magnetic permeability on strain. In addition, the equations are complemented with the usual mechanical condition of continuity of the total stress vector across the boundary, and the magnetic boundary conditions expressing the continuity of the magnetic scalar or vector potential and the normal component of the magnetic induction in the absence of surface electric currents:

\subsection{Equation of heat conduction}

The temperature $T$, as measured from a reference temperature $T_{0}$, satisfies the inhomogeneous Poisson's equation (c.f. [4] and [6]):

$$
\nabla^{2} T=-\frac{\mathrm{J}^{2}}{\mathrm{~K} \sigma}
$$

$\mathrm{K}$ is the coefficient of heat conduction and ${ }^{\sigma}$ the coefficient of electrical conductivity.

The general solution of equation (3) is

$$
\mathrm{T}=\mathrm{T}_{h}+\mathrm{T}_{\mathrm{p}^{\mathrm{s}}}
$$

where $T_{h}$ is the harmonic part of $T$ and $T_{p}$ is any particular solution of Poisson's equation (3). It is easily verified that

$$
\mathrm{T}_{\mathrm{p}}=-\frac{\mathrm{J}^{2}}{4 \mathrm{K \sigma} \sigma}\left(\mathrm{x}^{2}+\mathrm{y}^{2}\right)=-\frac{J^{2}}{4 K \sigma} \mathrm{r}^{2} .
$$

A Dirichlet thermal condition is taken at the boundary:

$$
\left(T_{h}+T_{p}\right)(\theta)=T_{e}(\theta) \text {. }
$$

The temperature distribution generates "temperature displacements", which will be included in the expressions for the mechanical displacement. These are [8]:

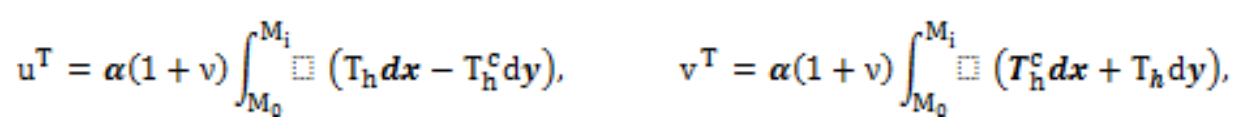

where $v$ and $\alpha$ are Poisson's ratio and the coefficient of linear thermal expansion respectively. Here $\mathrm{M}_{0} \in \overline{\mathrm{D}}$ is an arbitrarily chosen point in the domain of the cross-section, usually taken to coincide with 
the origin ${ }^{0}, \mathrm{M} \in \overline{\mathrm{D}}$ is the general point at which the function is calculated. The integration is path independent. Superscript 'c ', stands for the harmonic conjugate.

\subsection{Equations of Magnetostatics}

The magnetic vector potential is parallel to the axis of the cylinder (the ${ }^{z}$-axis), with component A . In the quasi-electrostatic approximation used here, it is well-known that the Cartesian components of the vector potential satisfy poisson's equation inside the matter. Thus (c.f. [4] and [6]):

$$
\nabla^{2} A=-\mu^{*} \mu_{0} J_{s}
$$

$\mu_{0}$ is the magnetic permeability of the cylinder and $\mu^{*}$ is the magnetic permeability of vacuum, with value $\mu^{*}=4 \pi \times 10^{-7} H \cdot \mathrm{m}^{-1}$.

In the surrounding vacuum labeled '*', one has $\mu_{0}=\mathbf{1}$ and $J=0$, so that

$$
\nabla^{2} A^{*}=0 \text {. }
$$

The solutions of equations (8), (9) are expressed as:

$$
\mathrm{A}=\mathrm{A}_{h}-\frac{1}{4} \mu^{*} \mu_{0} \mathrm{~J}\left(\mathrm{x}^{2}+\mathrm{y}^{2}\right), \quad \mathrm{A}^{*}=\mathrm{A}_{\infty}+\mathrm{A}_{\mathrm{r}}^{*}
$$

$A_{h}$ and $A_{h}^{*}$ being the harmonic parts of the vector potential inside and outside the domain $D$ respectively, and $\mathrm{A}_{\mathrm{mo}}$ is the expression for the vector potential far away from the cylinder's axis, giving the magnetic vector potential of a straight, infinite current-carrying wire, as may be verified in standard books of Electrodynamics, in the form:

$$
\mathrm{A}_{\mathrm{sc}}=-\frac{\mu^{*} \mathrm{~J} \mathrm{\Sigma}_{\mathrm{D}}}{2 \pi} \ln \frac{\mathrm{r}}{\mathrm{a}}, \quad \mathrm{\Sigma}_{\mathrm{D}} \text { is the cross - sectional area. }
$$

The boundary conditions for the magnetic problem illustrate the continuity of the tangential component of the magnetic field and the normal component of the magnetic induction. They are expressed as [8]:

$$
\begin{aligned}
& \frac{1}{\mu_{0}} \frac{\partial A_{h}}{\partial \tau}-\frac{\partial A_{r}^{*}}{\partial \tau}=\frac{\partial}{\partial \tau}\left(A_{m s}+\frac{1}{4} \mu^{*} J r^{2}\right), \\
& \frac{\partial A_{h}}{\partial \mathrm{n}}-\frac{\partial A_{r}^{*}}{\partial \mathrm{n}}=\frac{\partial}{\partial \mathrm{n}}\left(A_{m s}+\frac{1}{4} \mu^{*} \mu_{0} \mathrm{Jr}^{2}\right),
\end{aligned}
$$

and the vanishing behavior at infinity

$$
\mathrm{A}_{\mathrm{r}}^{*}(\mathrm{r} \rightarrow \infty)=\mathbf{0} .
$$

The magnetic field may be calculated from the magnetic vector potential using the well-known relation

$$
\mathrm{H}=\frac{1}{\mu^{*} \mu_{0}} \operatorname{curl} \mathrm{A}
$$

The magnetic field inside the conductor yields a distribution of "magnetic displacements", which will go into the equations for the mechanical displacement. These are: [8]:

$$
\mathrm{u}^{\mathrm{H}}=\int_{\mathrm{M}_{0}}^{\mathrm{M}_{\mathrm{i}}} \mathrm{E}\left(M_{\mathrm{H}} d \mathrm{x}+\mathrm{N}_{\mathrm{H}} d y\right), \quad \mathrm{v}^{\mathrm{H}}=\int_{\mathrm{M}_{0}}^{\mathrm{M}_{\mathrm{i}}} \mathrm{E}\left(\mathrm{R}_{\mathrm{H}} \mathrm{d} \boldsymbol{x}+S_{\mathrm{H}} d y\right),
$$


where

$$
\begin{array}{ll}
\mathrm{M}_{\mathrm{H}}=W\left(\mathrm{H}_{y}^{2}-\mathrm{H}_{\mathrm{x}}^{2}+\frac{\mathrm{J}^{2}}{2}\left(\mathrm{x}^{2}+\mathrm{y}^{2}\right)\right), & \mathrm{N}_{\mathrm{H}}=-2 \mathrm{~W}\left(\mathrm{H}_{x} \mathrm{H}_{y}+\frac{1}{\mu^{*} \mu_{0}} J \mathrm{~A}_{\mathrm{h}}^{\mathrm{c}}\right), \\
\mathrm{S}_{\mathrm{H}}=W\left(\mathrm{H}_{\mathrm{s}}^{2}-\mathrm{H}_{y}^{2}+\frac{\mathrm{J}^{2}}{2}\left(\mathrm{x}^{2}+\mathrm{y}^{2}\right)\right), & \mathrm{R}_{\mathrm{H}}=-2 W\left(\mathrm{H}_{x} \mathrm{H}_{y}-\frac{1}{\mu^{*} \mu_{0}} J \mathrm{~A}_{\mathrm{h}}^{\mathrm{c}}\right),
\end{array}
$$

and

$$
W=\frac{1}{2}(1+v) \frac{\mu^{*}}{E}\left(\mu_{0}-\frac{1}{2} \mu_{1}\right) .
$$

The integrations in (15) are path independent.

\subsection{Equations of Elasticity}

The basic equation is the biharmonic equation for the stress function $\mathrm{U}$

$$
\nabla^{4} U=\frac{\alpha \mathrm{E} \zeta}{(1-v) \mathrm{K}}-\frac{1-2 v}{2(1-v)} \mu^{*}\left(\frac{1}{2} \mu_{1}+\mu_{2}\right) \nabla^{2} H^{2}+\frac{\mu^{*} \mathrm{~J}^{2}}{1-v}\left(\mu_{0}-\frac{1}{2} \mu_{1}\right),
$$

of which the general solution is expressed in terms of two basic harmonic functions $\Phi_{s} \Psi$ as

$$
\mathrm{U}=\mathrm{x} \boldsymbol{\Phi}+\mathrm{y} \boldsymbol{\Phi}^{\mathrm{c}}+\Psi+\mathrm{U}_{\mathrm{p}}
$$

and $\mathrm{U}_{\mathrm{p}}$ is any particular solution of the equation:

$$
\nabla^{2} U_{\mathrm{p}}=-\frac{\alpha \mathrm{E}}{1-v} T_{\mathrm{p}}-\frac{1-2 v}{2(1-v)} \mu^{*}\left(\frac{1}{2} \mu_{1}+\mu_{2}\right) H^{2}+\frac{\mu^{*} \mathrm{~J}^{2}}{4(1-v)}\left(\mu_{0}-\frac{1}{2} \mu_{1}\right)\left(x^{2}+y^{2}\right) .
$$

The constants $\mu_{1}$ and $\mu_{2}$ appearing in the above formulae express the linear dependence of the magnetic permeability of the cylinder's material on strain as in [10], E is Young's modulus. the relations

The stress tensor components $\sigma_{\mathrm{xx}},{ }_{\mathrm{yy}}$ and ${ }^{\sigma_{\mathrm{xy}}}$ are defined through the stress function $\mathrm{U}$ by

$$
\sigma_{\mathrm{xx}}=\mathrm{U}_{3 \mathrm{yy}^{x}} \quad \sigma_{\mathrm{yy}}=\mathrm{U}_{\mathrm{szx}} \quad \sigma_{\mathrm{xy}}=-\mathrm{U}_{\mathrm{sxy}}
$$

where "comma" in the subscript means differentiation with respect to the shown variables.

The stress components are given in terms of $\phi$ and $\Psi$ as:

$$
\begin{aligned}
& \sigma_{\mathrm{xx}}=x \Phi_{y y}+2 \Phi_{y}^{\mathrm{c}}+\mathrm{y} \Phi_{y y}^{\mathrm{c}}+\Psi_{y y} \\
& \sigma_{\mathrm{xy}}=-x \Phi_{\mathrm{zxy}}-\mathrm{y} \Phi_{\mathrm{zxy}}^{\mathrm{c}}-\Psi_{\mathrm{zxy}} \\
& \sigma_{\mathrm{yy}}=\boldsymbol{x} \Phi_{x \mathrm{xx}}+2 \boldsymbol{\Phi}_{x}+\mathrm{y} \Phi_{z \mathrm{xx}}^{\mathrm{c}}+\boldsymbol{\Psi}_{x x}
\end{aligned}
$$

The mechanical displacement in Cartesian coordinates are expressed as:

$$
\begin{aligned}
& \frac{\mathrm{E}}{1+v} \mathrm{u}=-\mathrm{U}_{y \mathrm{x}}+4(1-\mathrm{v}) \Phi+\frac{\mathrm{E}}{1+v}\left(\mathrm{u}^{\mathrm{T}}+\mathrm{u}^{H}\right), \\
& \frac{\mathrm{E}}{1+v} \mathrm{v}=-\mathrm{U}_{y y}+4(1-v) \Phi^{c}+\frac{\mathrm{E}}{1+v}\left(\mathrm{v}^{T}+\mathrm{v}^{\mathrm{H}}\right),
\end{aligned}
$$

where $\mathrm{u}^{\mathrm{T}}, \mathrm{v}^{\mathrm{T}}$ and $\mathrm{u}^{\mathrm{H}}, \mathrm{v}^{\mathrm{H}}$ are defined in (7), (15). 
The tangential component $\boldsymbol{u}_{\tau}$ and the normal component $u_{n}$ of the mechanical displacement at the boundary are sometimes needed as boundary conditions. They read:

$$
\begin{aligned}
& \frac{\mathrm{E}}{1+v} \mathrm{u}_{\tau}=(3-4 v)\left(\frac{\dot{\mathrm{x}}}{\omega} \Phi+\frac{\dot{\mathrm{y}}}{\omega} \Phi^{\mathrm{c}}\right)-\frac{x}{\omega} \dot{\Phi}-\frac{\mathrm{y}}{\omega} \Phi^{c}-\frac{1}{\omega} \dot{\Psi} \\
& -\frac{\dot{\mathrm{x}}}{\omega} \mathrm{U}_{\mathrm{p}, \mathrm{x}}-\frac{\dot{\mathrm{y}}}{\omega} \mathrm{U}_{\mathrm{p}, y}+\frac{\mathrm{E}}{1+v}\left(\frac{\dot{\mathrm{x}}}{\omega} \boldsymbol{u}^{\mathrm{T}}+\frac{\dot{\mathrm{y}}}{\omega} \mathrm{v}^{T}\right) . \\
& \frac{\mathrm{E}}{1+v} \mathrm{u}_{\mathrm{n}}=(3-4 v)\left(\frac{\dot{\mathrm{y}}}{\omega} \Phi-\frac{\dot{\mathrm{x}}}{\omega} \Phi^{\mathrm{c}}\right)+\frac{y}{\omega} \dot{\Phi}-\frac{\mathrm{x}}{\omega} \Phi^{c}-\frac{1}{\omega} \dot{\bar{c}} \\
& -\frac{\dot{\mathrm{y}}}{\omega} \mathrm{U}_{\mathrm{p}, \mathrm{x}}+\frac{\dot{\mathrm{x}}}{\omega} \mathrm{U}_{\mathrm{p}, y}+\frac{\mathrm{E}}{1+v}\left(\frac{\dot{\mathrm{y}}}{\omega} \boldsymbol{u}^{\mathrm{T}}-\frac{\dot{\mathrm{x}}}{\omega} \mathrm{v}^{T}\right) .
\end{aligned}
$$

The boundary conditions associated with the mechanical problem specify a uniform normal extension of value $\epsilon=0.1$. Other types of mechanical conditions may be treated equally well. The rigid body motion is eliminated as usual. Details may be found in [13].

\section{THE METHOD OF SOLUTION}

\subsection{Generation of harmonic functions}

Our problem involves five harmonic functions, related by boundary conditions, to be determined: Functions $T_{h}$ for the temperature, $A_{h}, A_{r}^{*}$ for the magnetic vector potential in the whole space, and $\boldsymbol{\Phi}$, $\Psi$ for the stress function. These functions will be expanded in terms of Cartesian polynomial and rational harmonics in a way explained in [13]. These harmonic functions contain a number of coefficients, to be determined by the method of boundary collocation to satisfy the boundary conditions of the problem.

\subsection{Solution to the inhomogeneous biharmonic equation}

There is a permanent interest in resolving Poisson's equation and the non-homogeneous biharmonic equation in two-dimensional regular or irregular regions (c.f. [16, 17]). The proposed method yields an easy resolution of the involved non-homogeneous biharmonic equation (18), independently of the complexity of the r.h.s. of this biharmonic equation. Due to the expansions of the harmonic functions representing the solution, the r.h.s. of the biharmonic equation turns out to be a polynomial expression in $\mathrm{x}$ and $\mathrm{y}$. Use of the expressions

$$
x=\frac{z+\bar{z}}{2}, \quad y=\frac{z-\bar{z}}{2 i}
$$

and the fact that

$$
\nabla^{2}=2 \frac{\partial}{\partial z} \frac{\partial}{\partial z}
$$

makes it possible to directly integrate the inhomogeneous biharmonic equation for the particular solution. Once the integration is completed, the variables $x$ and $y$ are restored back into the obtained expression for the particular solution $\mathrm{U}_{\mathrm{p}}$.

\subsection{Coefficients of the expansions}

The coefficients of the expansions of the different harmonic functions are determined by collocation through the satisfaction of: (i) the boundary conditions, (ii) the conditions at the interface, (iii) the conditions for eliminating the rigid body motion at the origin of coordinates, (iv) the 
additional conditions at particular points of the boundary. This requires a choice of some boundary points at which the boundary conditions are exactly satisfied. As a result, one obtains a system of linear algebraic equations for the determination of the unknown coefficients. Finally, all quantities of practical interest are determined everywhere in $\mathrm{D}$ and in $\mathrm{C}(\overline{\mathrm{D}})$.

\subsection{Error estimation}

The field equations are satisfied rigorously. The only errors in the solution are those arising from the solutions of three systems of linear algebraic equations satisfying the thermal and the mechanical boundary conditions, and the interfacial conditions of the magnetic field. Any of these rectangular systems of linear algebraic equation in matrix form

$$
\mathrm{AX}=\boldsymbol{B},
$$

is reduced to a square system by the transformation

$$
\left(\mathrm{A}^{\mathrm{T}} \mathrm{A}\right) \boldsymbol{X}=\mathrm{A}^{\mathrm{T}} \boldsymbol{B},
$$

where superscript $\mathrm{T}$ denotes the transpose. The solution then follows by Least Squares. If $\mathrm{Z}$ denotes the obtained solution of this last system, the error in satisfying the system of equations is defined as

$$
\mathrm{ER}=\max _{\overline{\mathrm{i}}}\left|\mathrm{A}_{\mathrm{ij}} \mathrm{Z}_{\mathrm{j}}-\mathrm{B}_{\mathrm{i}}\right| \text {. }
$$

\section{APPLICATION AND NUMERICAL RESULTS}

The application considered here below concerns the so-called nearly-circular contour with parametric equations:

$$
\mathrm{x}=\mathrm{a}(1+\epsilon \cos \theta) \cos \theta, \quad \mathrm{y}=\mathrm{a}(1+\epsilon \cos \theta) \sin \theta,
$$

where $\mathbf{a}$ and $\boldsymbol{\epsilon}$ are positive geometric parameters. In what follows, we choose $\mathrm{a}=1$. When $€=0$, the contour becomes circular with unit radius. As $\boldsymbol{\in} \rightarrow \mathbf{1}$, the contour tends to a cardioid. Fig.2 shows a nearly-circular contour with $\epsilon=0.5$, in comparison to a circular boundary of unit radius. The almost flat part of the boundary is centered around the boundary point $\theta=\pi$ on the interval $[\pi-0.5314, \pi+0.5314]$ approximately. The normal cross-sectional area of the cylinder is $\mathrm{z}_{\mathrm{D}} \simeq 3.534$, which is a bit larger than the area of the unit circle.

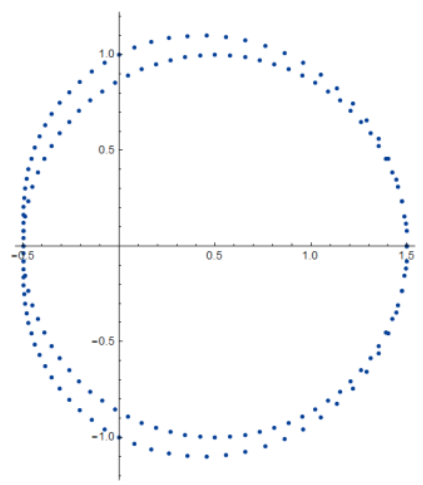

Figure 2: A nearly-circular contour with $\epsilon=0.5$ (solid contour), compared to a circular boundary of unit radius (dotted contour) with two points of tangency. Notice the flat part to the left.

All the equations are expressed in dimensionless form. For this purpose, introduce the dimensionless quantities: 


$$
\begin{aligned}
& \mathrm{x}^{\prime}=\frac{\mathrm{x}}{a^{v}}, \quad y^{\prime}=\frac{\mathrm{y}}{\mathrm{a}}, \quad \mathrm{u}^{\prime}=\frac{\mathrm{u}}{\mathrm{a}(1+\mathrm{v})}, \quad \boldsymbol{v}^{\prime}=\frac{\mathrm{v}}{\mathrm{a}(1+\mathrm{v})}, \quad \mathrm{T}^{\prime}=\alpha T_{v} \\
& \mathrm{~A}^{s}=\frac{\mathrm{A}}{\mu^{*} \mathrm{~J} \mathrm{a}^{2}}, \quad \mathrm{H}_{\mathrm{x}}^{s}=\frac{H_{\mathrm{x}}}{\mathrm{a} J}, \quad \mathrm{H}_{\mathrm{y}}^{s}=\frac{\mathrm{H}_{\mathrm{y}}}{\mathrm{a} J}, \\
& \mathrm{U}^{\prime}=\frac{\mathrm{U}}{\mathrm{a}^{2} \mathrm{E}}, \quad \sigma_{\mathrm{xx}}^{\prime}=\frac{\sigma_{\mathrm{xx}}}{\mathrm{E}}, \quad \sigma_{\mathrm{yy}}^{\prime}=\frac{\sigma_{\mathrm{yy}}}{\mathrm{E}}, \quad \sigma_{\mathrm{xy}}^{\prime}=\frac{\sigma_{\mathrm{xy}}}{\mathrm{E}},
\end{aligned}
$$

and two dimensionless parameters:

$$
\beta=\frac{\alpha \mathrm{a}^{2} \mathrm{~J}^{2}}{4 \mathrm{~K} \sigma}, \quad \gamma=\frac{4 \mu^{*} \mathrm{~K} \sigma}{\alpha \mathrm{E}} .
$$

For definiteness, calculations have been carried out with collocation nodes uniformly distributed w.r.t. the central polar angle on the contour. The values of the different dimensionless material parameters were taken as:

$$
\mu_{0}=0.99, \quad \mu_{1}=0.20, \quad \mu_{2}=0.25, \quad v=0.25, \quad \beta=0.2, \quad \gamma=0.05 .
$$

A Dirichlet thermal condition with varying temperature, together with a uniform normal extension, are prescribed on the boundary. Numerous numerical experiments have been carried out for different expansions and different numbers of collocation nodes, only the best results have been retained. Additionally, improvements can be achieved by varying the number and the positions of the nodes on the boundary. This latter aspect has been disregarded for the sake of conciseness.

The distribution of temperature prescribed on the boundary is in the form:

$$
\mathrm{T}_{\mathrm{e}}(\theta)=0.01(1+\cos 2 \theta)
$$

and a normal extension of value $\epsilon=0.10$ is applied to the boundary.

The number of terms for the different expansions that yielded the present results were as follows: 20 terms for the temperature, 10 terms for each of the internal and the external vector potential, 20 terms for the elasticity harmonic functions, and $\mathbf{8 0}$ collocation nodes. The absolute errors in satisfying the arising three linear systems of algebraic equations did not exceed 0.0036 .

The following figures show the comparison between the nearly-circular and the circular contours in the boundary distributions of some functions involved in the solution.

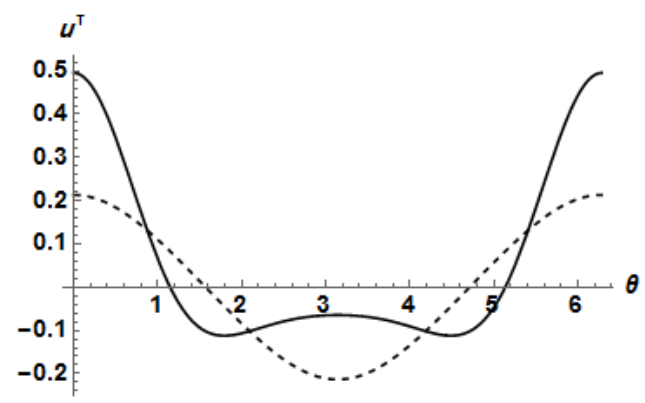

Figure 3: The boundary temperature displacement ${ }^{\mathrm{u}^{\mathrm{T}}}$ (solid) compared to the circular case (dotted). 


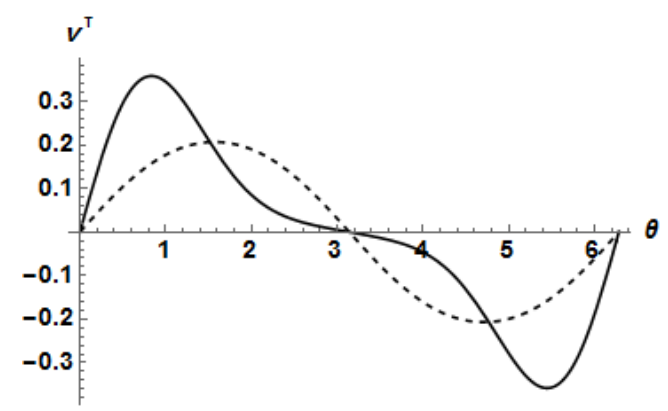

Figure 4: The boundary temperature displacement $\mathrm{v}^{\mathrm{T}}$ (solid) compared to the circular case (dotted).

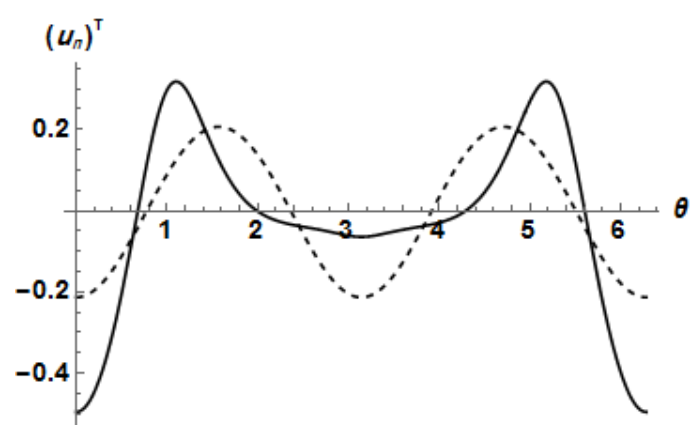

Figure 5: The normal boundary temperature displacement $\mathrm{u}_{\mathrm{n}}^{\mathrm{T}}$ (solid) compared to the circular case (dotted).

The components of the magnetic field and the stress at boundary points can be measured. The form of this boundary will undoubtedly affect the values of the different components of the magnetic field and the stress. It is thus possible to detect the imperfections in the boundary shape by purely magnetic measurements, and also by mechanical measurements involving the stress. For boundary conditions involving the stress, one could measure the boundary displacement.

Both components of the magnetic field on the boundary vary in the approximate range $[-0.5,0.5]$. The amplitudes of variation for the nearly-circular contour are somewhat larger than the corresponding quantities for the circular contour as in figs. 6 and 7. This is due to the difference in area between the two contours. The boundary component $\mathrm{H}_{\mathrm{s}}$ of the magnetic field parallel to the axis of symmetry of the contour has a point of inflection at the center of the flat part with tangent inclined at a smaller angle to the axis than for the circular case. As seen in fig.7, the boundary component $\mathrm{H}_{y}$ of the magnetic field perpendicular to the axis of symmetry of the contour along the flat part of the boundary is almost constant and is at a minimum. This is to be compared with the local minimum for the circular boundary at almost the same value. The maximum value of this component is attained on the axis of symmetry of the contour on the opposite side. The normal component of the magnetic field at the boundary is shown in fig. 8 .

The normal stress distribution on the boundary is shown in fig. 12 for both contours. Due to the applied boundary displacement, it may be noticed that the normal stress for the circular contour amounts to a compression at all points of the contour. This is not the case for the nearly-circular contour, where parts of the boundary acquire positive values. Moreover, the amplitude variation of the normal stress for the nearly-circular contour is almost two-thirds of its value for the circular contour. 


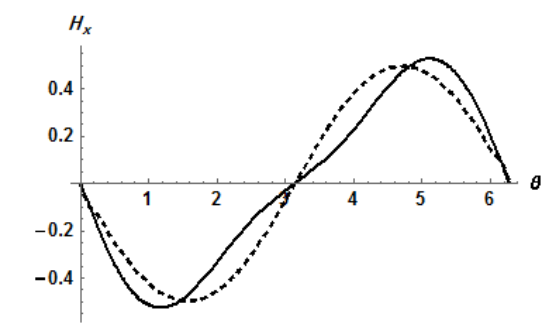

Figure 6: The boundary magnetic field component $\mathrm{H}_{\mathrm{K}}$ (solid) compared to the circular case (dotted).

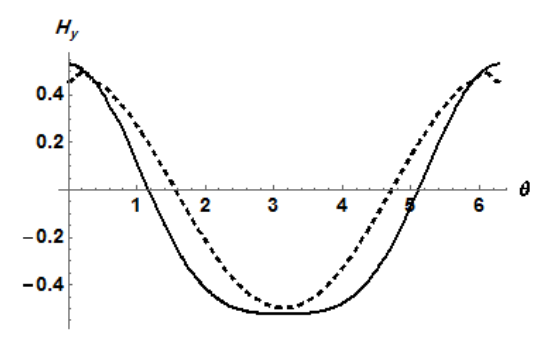

Figure 7: The boundary magnetic field component ${ }^{\mathrm{H}_{y}}$ (solid) compared to the circular case (dotted).

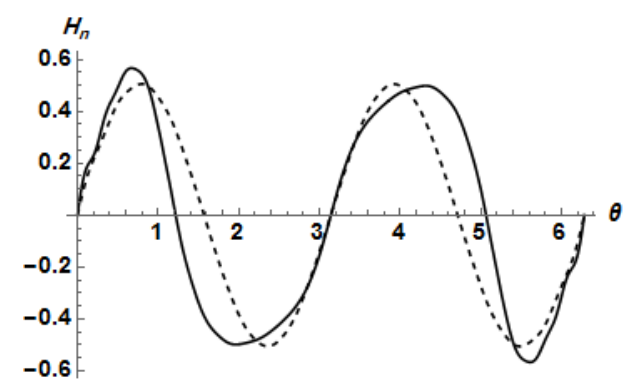

Figure 8: The boundary normal magnetic field $\mathrm{H}_{\mathrm{n}}$ (solid) compared to the circular case (dotted).

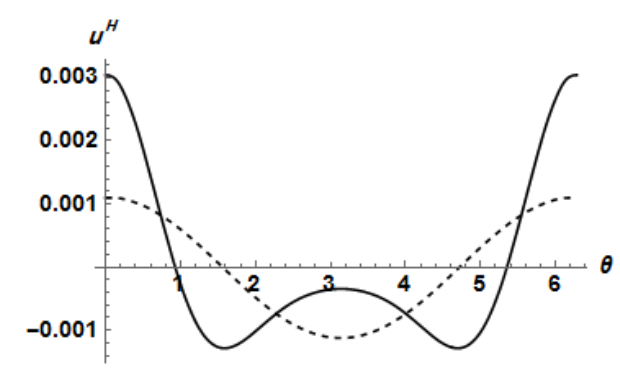

Figure 9: The boundary magnetic displacement $\mathrm{u}^{\mathrm{H}}$ (solid) compared to the circular case (dotted).

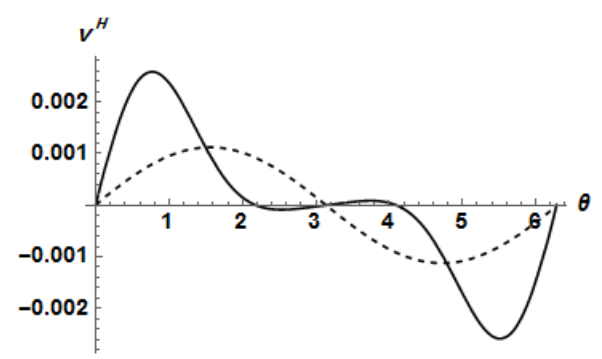

Figure 10: The boundary magnetic displacement $\mathrm{v}^{\mathrm{H}}$ (solid) compared to the circular case (dotted). 


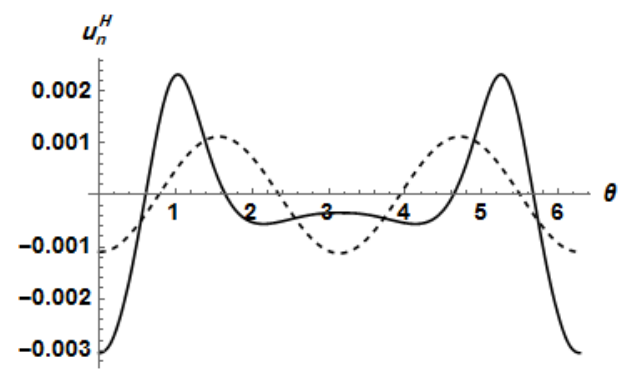

Figure 11: The boundary normal magnetic displacement $\mathrm{u}_{\mathrm{n}}^{\mathrm{H}}$ (solid) compared to the circular case (dotted).

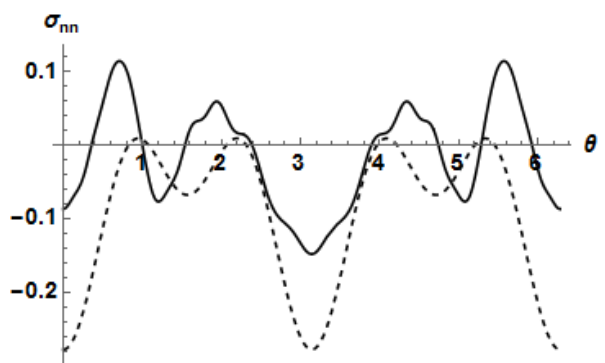

Figure 12: The boundary normal stress component $\sigma_{\mathrm{nn}}$ (solid) compared to the circular case (dotted).

\section{EFFECT OF THE MAGNETIC PERMEABILITY DEPENDENCE ON STRAIN}

The present model foresees the dependence of the magnetic permeability tensor on strain as:

$$
\mu_{\mathrm{ij}}=\mu_{0} \delta_{\mathrm{ij}}+\mu_{1} \epsilon_{\mathrm{if}}+\mu_{2} I_{1} \delta_{\mathrm{iff}}
$$

where $\epsilon_{\mathrm{ij}}$ denote the strain tensor components and $\mathrm{I}_{\mathbf{1}}$ is the first invariant of this tensor (c.f. [4]). The two material parameters $\mu_{1}$ and $\mu_{2}$ illustrating the dependence of the magnetic permeability on strain are not found in the literature. One of the aims of the present work is to use the obtained approximate solution to propose ways for finding the values of these two parameters. Figure13 illustrates the boundary normal stress for two values of the parameter $\mu_{1}$ and for $\mu_{2}=0.25$. Taking in consideration that the boundary is subjected to a uniform extension $\epsilon=0.1$, an increase of the value of parameter $\mu_{1}$ increases the values of the normal stress to become all positive.

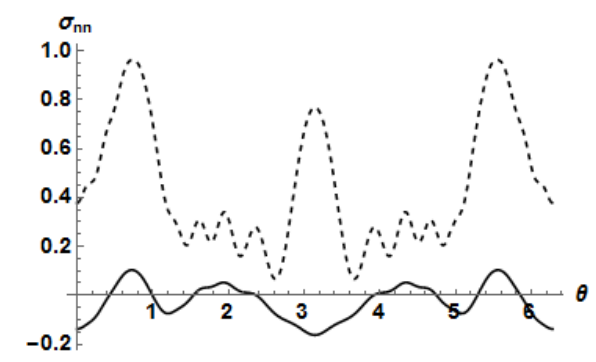

Figure 13: The boundary normal stress component $\sigma_{\mathrm{mn}}$ for $\mu_{1}=0.2$ (solid) and for $\mu_{1}=100.0$ (dotted).

Figure14 shows that the effect of the increase of parameter $\mu_{2}$ is to decrease the values of the normal stress. Thus, the increase of the two parameters $\mu_{1}$ and $\mu_{2}$ yields the opposite effects. 


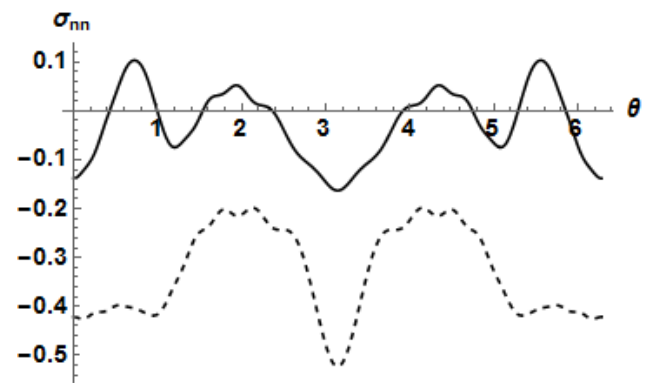

Figure 14: The boundary normal stress component $\sigma_{\operatorname{mn}}$ for $\mu_{2}=0.25$ (solid) and for $\mu_{2}=100.0$ (dotted).

\section{CONCLUSIONS}

A method of solution previously proposed by the authors has been applied to the plane problem of thermo-magnetoelasticity of a long cylinder with nearly-circular normal cross-section carrying a steady, uniform electric current in the quasi-electrostatic approximation. The boundary is subjected to a Dirichlet-type thermal boundary condition, in addition to a prescribed boundary normal mechanical displacement.

Comparison with the circular contour has allowed us to assess the influence of the boundary shape imperfection on the distribution of the magnetic field and normal stress on the boundary. Measurement of the boundary magnetic field allows estimating the degree of imperfection of the boundary shape, i.e its deviation from the circular boundary. This can also be achieved by measurement of the normal stress component.

\section{REFERENCES}

[1] G.A. Maugin Continuum mechanics of electromagnetic solids, in North-Holland series in Applied Mathematics and Mechanics, eds. J.D. Achenbach, B. Budiansky, W.T. Koiter, H.A. Lauwerier, L. van Vijngaarden, 33, North-Holland Publishing Company, North Holland, Amsterdam, new York, Oxford, Tokyo (1988).

[2] A.C. Eringen, G.A. Maugin Electrodynamics of continua, vol. I, Springer-Verlag, New York, Berlin, Heidelberg, London, Paris, Tokyo, Hong Kong (1989); Electrodynamics of continua, vol. II, SpringerVerlag, New York, Berlin, Heidelberg, London, Paris, Tokyo, Hong Kong (1990)

[3] R.W. Ogden and D. J. Steigmann, Eds., Mechanics and electrodynamics of magneto- and electro-elastic materials, CISM Courses and lectures 527, Springer, Wien New York (2011).

[4] A. F. Ghaleb, Deformation of an infinite, hollow circular cylindrical conductor carrying a current, Proc. Math. Phys. Soc. Egypt 45, 63-69 (1978).

[5] M. M. Ayad, A. A. Mosharafa, H. A. Z. Hassan, A. F. Ghaleb, Deformation of an infinite elliptic cylindrical conductor carrying a uniform axial current, Mechanics of Materials 17, 351-361 (1994).

[6] M.S. Abou-Dina, A.F. Ghaleb, On the boundary integral formulation of the plane Theory of Thermomagnetoelasticity, Int. J. Appl. Electromagn. Mech. 11, 185-201 (2000).

[7] E. A. Deviatkin, Lorentz force-induced stresses in long current-carrying conductors, Int. J. Appl. Electromagn. and Mech. 16, 99-110 (2002). 
[8] A. N. El Dhaba, M. S. Abou-Dina, A. F. Ghaleb, A plane problem of uncoupled thermomagnetoelasticity for an infinite, elliptical cylinder carrying a steady axial current by a boundary integral method, Appl. Math. Mod. 31, 448-477 (2007).

[9] A. R. El Dhaba, A. F. Ghaleb, M. S. Abou-Dina, Deformation of a long, current-carrying elastic cylinder of square cross-section: numerical solution by boundary integrals, Arch. Appl. Mech. 84, 1393-1407 (2014). DOI 10.1007/s00419-014-0857-7

[10] A. R. El Dhaba, A. F. Ghaleb, J. El-Seadawy, M. S. Abou-Dina, Uncoupled thermomagnetoelastostatics for long cylinders carrying a steady axial electric current by a boundary integral method. A numerical approach, Int. J. Appl. Electromagn. Mech. 46, 629-648 (2014). DOI 10.3233/JAE-141961

[11] A. R. El Dhaba, Deformation of an infinite, square rod of an elastic magnetizable material, subjected to an external magnetic field by a boundary integral method: a numerical approach, J. Vibr. Control 22, 16, 3532-3543 (2016).

[12] A. R. El Dhaba, A. F. Ghaleb, Deformation of an elastic magnetizable square rod due to a uniform electric current inside the rod and an external transverse magnetic field, Math. Mech. Solids 21, 2, 222-241 (2016).

[13] S. M. El Sheshtawy, A.-S. F. Obada, M. S. Abou-Dina, A. F. Ghaleb, Semi-analytical solution by Cartesian harmonic polynomials for a problem of deformation of a long, current-carrying elastic cylindrical conductor, Math. Mech. Solids, 22 pages. DOI: 10.1177/1081286520916221

[14] M.I.A. Othman, I.A. Abbas, Effect of rotation on a magneto-thermoelastic hollow cylinder with energy dissipation using finite element method, J. Comput. Theoret. Nanosci., 12(9) 2399-2404 (2015).

[15] H. Altenbach, O. Morachkovsky, K. Naumenko, D. Lavinsky, Inelastic deformation of conductive bodies in electromagnetic fields, Cont. Mech. Thermodyn. 28, 1421-1433 (2016). DOI 10.1007/s00161-015-0484-8

[16] I. K. Youssef, Sh. A. Meligy, Boundary-value problems on triangular domains and MKSOR methods, Appl. Comp.1 Math. 3, 3, 90-99 (2014). doi: 10.11648/j.acm.20140303.14

[17] V. A. Belyaev, V. P. Shapeev, Solving the biharmonic equation in irregular domains by the least squares collocation method, AIP Conference Proceedings 2027, 030094 (2018). doi.org/10.1063/1.5065188 


\section{الحلول التقريبية لمسألة مرور تيار كهربى فى مهى \\ إسطوانة مرنة طويلة المقطع على شكل أشباه دوائر بطريقة دوال كثيرات الحدود الكارتيزية}

سيا محمد الششتاوى 1 , عبدالثافى فهمى عبادة 1 , مصطفى صابر أبودينه 2, , أحمد فؤاد غالب 2 1 - قسم الرياضيات , كلية العلوم (بنين) , جامعة الأزهر , مدينة نصر, القاهرة , مصر

2 - قسم الرياضيات , كلية العلوم , جامعة القاهرة , الجيزة , مصر , رلئر

\section{ملخص البحث:}

فى هذا البحث قمنا بحل مسألة نشوه موصل كهربائى طويل مقطعه العمودى شبه دائرى، يحمل تيارا كهربائيا محوريا ثابتا تحت تأثير حرارة "جول" وشرط دريشليه الحرارى على السطح، وشرط ازاحة ميكانيكية عمودية منتظمة

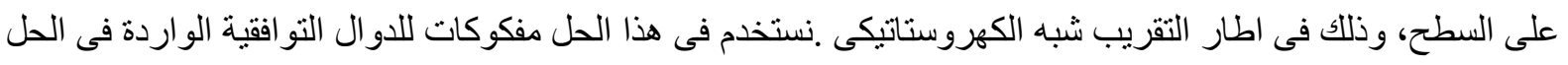
بدلالة دوال كثير ات حدود ودو ال كسرية نو افقية ديكارتبة. حصلنا على نتائج عددية وقمنا برسم الكمبات ذات الاهمية

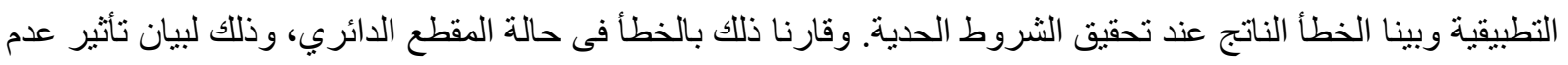

